\title{
The alpha subunit of Go modulates cell proliferation and differentiation through interactions with Necdin
}

\author{
Hyunhee Ju' ${ }^{1 \dagger}$, Sujin Lee ${ }^{1 \dagger}$, Sunghak Kang ${ }^{1 \dagger}$, Sung-Soo Kim² and Sungho Ghil ${ }^{1 *}$
}

\begin{abstract}
Background: Heterotrimeric GTP-binding proteins (G-proteins) play an important role in mediating signal transduction generated by neurotransmitters or hormones. Go, a member of the Gi/Go subfamily, is the most abundant G-protein found in the brain. Recently, the alpha subunit of $\mathrm{Go}(\mathrm{GaO})$ was characterized as an inducer of neuronal differentiation. However, its underlying molecular mechanisms have remained unclear to date, since the downstream effectors of Gao are ambiguous.

Results: A neurally differentiated embryonal carcinoma-derived protein (Necdin) was isolated as an interacting partner for Gao from a mouse brain cDNA library using yeast two-hybrid screening. Interactions between the proteins were confirmed with several affinity binding assays, both in vitro and in vivo. Necdin interacted directly and preferentially with activated Gao, compared to wild-type protein. Interestingly, Gao did not interact with Gai, despite high sequence homology between the two proteins. We subsequently analyzed whether Gao modulates the cellular activities of Necdin. Notably, expression of Gao significantly augmented Necdin-mediated cellular responses, such as proliferation and differentiation. Moreover, activation of type 1 cannabinoid receptor (CB1R), a Gi/oa-coupled receptor, augmented cell growth suppression, which was mediated by Gao and Necdin in U87MG cells containing CB1R, Gao, and Necdin as normal components.
\end{abstract}

Conclusions: These results collectively suggest that Necdin is a candidate downstream effector for Gao. Our findings provide novel insights into the cellular roles of Gao and its coupled receptor.

Keywords: Cannabinoid, E2F1, G-protein coupled receptor, Yeast two-hybrid

\section{Background}

Heterotrimeric GTP-binding proteins (G-proteins) mediate signaling from $\mathrm{G}$ protein-coupled receptors (GPCRs) to intracellular downstream effectors [1]. Binding of agonists to GPCR stimulates G-protein activation by inducing guanine nucleotide exchange from GDP to GTP. This facilitates dissociation of the alpha subunit $(\mathrm{G \alpha})$ from beta/gamma subunits $(\mathrm{G} \beta \gamma)$ of G-protein. Dissociated G-protein subunits, in turn, modulate activation of their downstream effectors.

To date, $21 \mathrm{G \alpha}$ subunits encoded by 16 genes have been identified [2]. Goo has been classified as a member

\footnotetext{
* Correspondence: shghil@kgu.ac.kr

${ }^{\dagger}$ Equal contributors

'Department of Life Science, Kyonggi University, Suwon 443-760, Republic of Korea

Full list of author information is available at the end of the article
}

of the Gi/o family, owing to its sequence homology with Goi, and is the most abundant Go protein in brain tissue. Overexpression of Goo promotes neuronal differentiation in various cell types, including PC12, N1E115, and Neuro2a [3-5]. Previously, we demonstrated that Goo increases the number of newly forming neurites in an F11 neuroblastoma cell line [6]. Goo additionally induces activation of Ras-like protein in all tissues (Rit), which triggers Erk-mediated neuronal differentiation in neuro2A cells [7]. These findings collectively indicate that Goo acts as an inducer of neuronal differentiation in neurogenic cells. However, the downstream effectors for Goo and related signaling pathways have not been fully elucidated.

Cannabinoids, the major components of Cannabis sativa Linnaeus (marijuana), have recently received considerable attention as potential therapeutic agents, owing to their various pharmacological actions, including pain control, 
tumor regression, neurogenesis, neuroprotection, and antiinflammatory effects [8-12]. Two types of cannabinoid receptors, designated $\mathrm{Gi} / \mathrm{o}$-coupled receptors, have been identified: (1) type I cannabinoid receptor (CB1R) cloned in 1990 [13], predominantly expressed in the brain [14], and (2) type II cannabinoid receptor (CB2R) cloned in 1993 [15], mainly expressed in cells of the immune system [16].

Neurally differentiated embryonal carcinoma-derived protein (Necdin) was originally isolated from P19 embryonic carcinoma cells [17]. Necdin, primarily identified as a functional analog of retinoblastoma protein (Rb), acts as a cell growth suppressor [18]. Additionally, Necdin is reported to induce differentiation in various cell types, including neuronal, muscular, and adipose cells [17,19-21]. Necdin interacts with several Rb-interacting proteins, including SV40 large $\mathrm{T}$ antigen and adenovirus E1A, and binds directly to the transcription factor, E2F1, to inhibit its function [22]. Similar to Rb, which induces neuronal differentiation by inhibiting E2F1-associated cell cycle progression, ectopic expression of Necdin triggers neuronal differentiation in N1E-115 neuroblastoma cells [23].

In this study, we performed yeast two-hybrid screening to identify downstream effectors for Goo using a constitutively active form of Goo as bait from a mouse brain cDNA library. Consequently, Necdin was identified as a Goo-interacting protein. Interactions between Goo and Necdin, both in vitro and in vivo, were further confirmed with several affinity binding assays. Furthermore, activation of Goo enhanced Necdin activity. Our findings collectively indicate that Necdin is a candidate downstream effector for Goo.

\section{Results}

Interactions of Gao with Necdin in vitro and in vivo

We performed yeast two-hybrid screening to identify Goo-interacting partners from a mouse brain cDNA library. Necdin protein was isolated using the constitutively active mutant of Goo as bait, and interactions confirmed with several affinity binding assays. Beads charged with bacterially expressed GST or GST-Goo proteins were incubated with soluble proteins obtained by detergent extraction of 293T cells transfected with FLAG-tagged Necdin (FLAG-Necdin), and the reaction mixtures probed with antibodies against FLAG. GST-Goo specifically interacted with FLAG-Necdin (Figure 1A). To determine whether these interactions also occur in the mammalian cellular context, 293T cells were transfected with plasmids encoding wild type of Goo (GooWT) and FLAG-Necdin, and the lysates immunoprecipitated and immunoblotted with the indicated antibodies. Consistently, Necdin was identified as an interacting partner of Goo in mammalian cells (Figure 1B). To investigate whether the two proteins co-localized in 293T cells, cells were transfected with plasmids encoding GooWT and FLAG-Necdin, and stained with the corresponding antibodies. Confocal microscopy images obtained from the immunofluorescence study indicated co-localization of the two proteins in the cell membrane (Figure 1C). Immunoprecipitation experiments were performed using newborn rat brain extracts to investigate endogenous interactions between the two proteins. Immunocomplexes that precipitated with antibodies against Goo were analyzed using anti-Necdin antibodies. Necdin proteins were detected in immunoprecipitates of Goo (Figure 1D). Finally, we performed immunohistochemistry using mouse E15.5 brain to test their expression and co-localization in vivo. As shown in Figure 1E, Goo was mainly expressed in cell membrane and cell process of subplate neuron. Necdin was also expressed in perinuclear region of both cortical plate and subplate neurons. Goo and Necdin were colocalized in cell membrane of subplate neuron. These results clearly imply that Goo specifically interacts with Necdin in vitro as well as in vivo.

\section{Characteristics of Gao interactions with Necdin}

To determine whether Necdin functions as a downstream effector for Goo, we incubated with purified His-Goo and/or glutathione-S-transferase (GST)-tagged Necdin (GST-Necdin) proteins in the presence or absence of $\mathrm{AlF}_{4}^{-}$, an activator of $\mathrm{G} \alpha$ protein. After glutathioneSepharose beads were added to the reactions, beads were analyzed with antibodies against Goo and Necdin. Necdin bound directly to Goo in the presence of $\mathrm{AlF}_{4}^{-}$(Figure 2A). Next, we performed a co-immunoprecipitation assay using lysates of $293 \mathrm{~T}$ cells expressing plasmids encoding the wild-type and constitutively active form of Goo, together with FLAG-Necdin. Immunocomplexes precipitating with anti-Goo antibodies were analyzed with immunoblot analysis using antibodies against FLAG and Goo. Notably, the constitutively active form of Goo showed higher affinity for Necdin than the wild-type protein (Figure 2B). In view of these results showing that Necdin binds directly and preferentially to the activated form of Goo, we propose that Necdin is a candidate downstream effector for Goo.

Since Goo displays a high degree of sequence homology

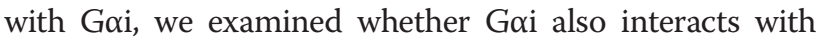
Necdin using lysates of $293 \mathrm{~T}$ cells co-expressing either

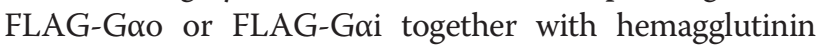
(HA)-tagged-Necdin (HA-Necdin). Cell lysates were incubated with antibodies against Necdin, and the immunocomplexes analyzed with immunoblot analysis using the indicated antibodies (Figure 3B). Interestingly, however, Goi did not interact with Necdin, unlike Goo. Next,

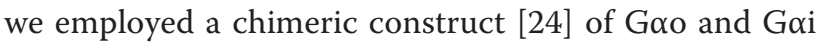
to investigate the Necdin-interacting domain in Goo (Figure 3A). Lysates of 293T cells co-expressing plasmids encoding FLAG-G $\alpha$ chimeric proteins and HA-Necdin were subjected to immunoprecipitation analysis with 


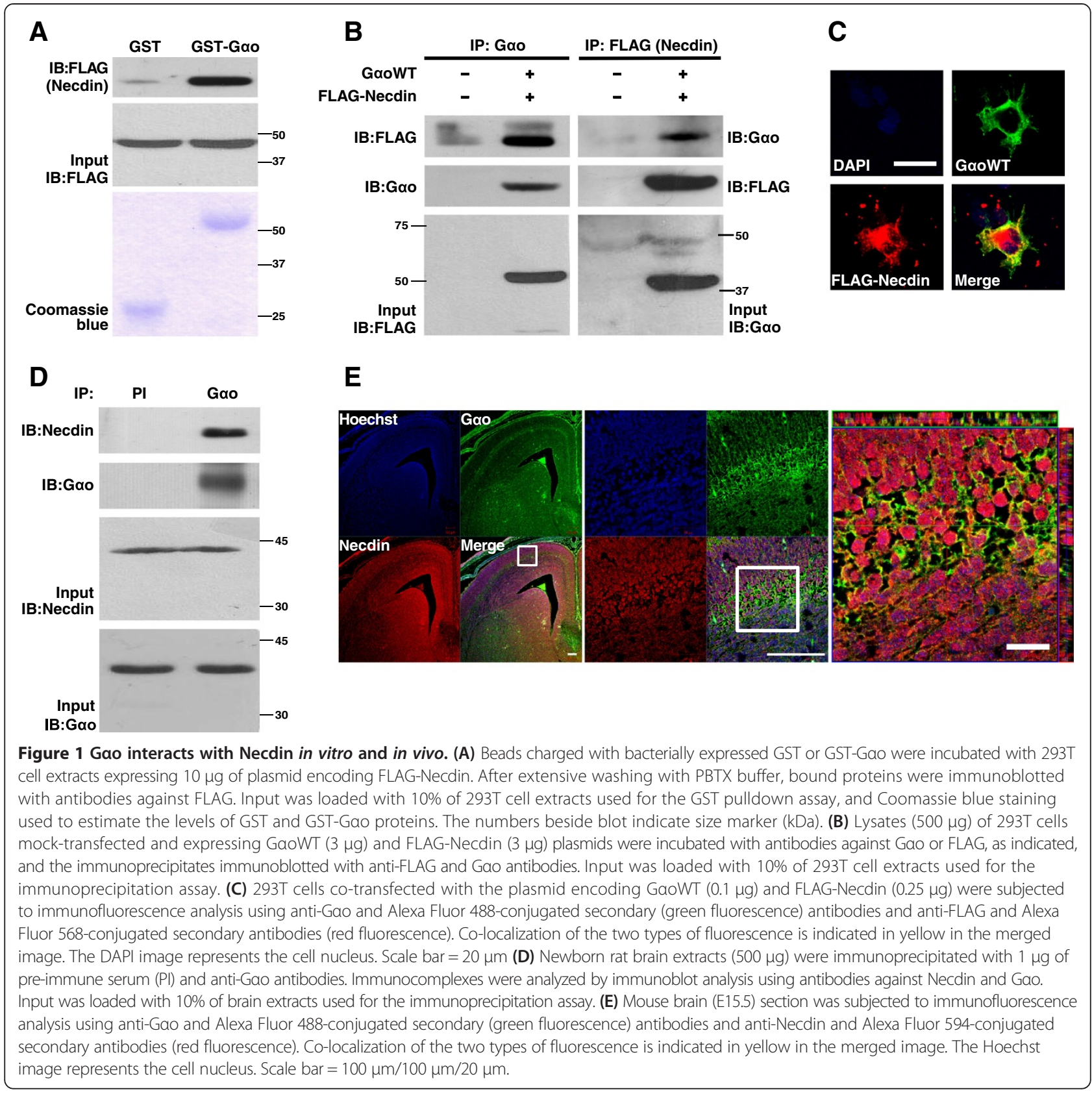

antibodies against Necdin. A chimeric protein, Gai/o,

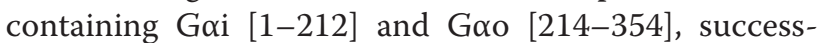

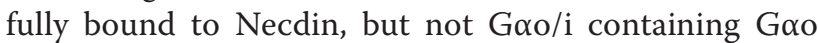
[1-213] and Gai [213-354] (Figure 3B). Our results suggest that Goo interacts with Necdin via part of the C-terminal region required for GTPase activity.

\section{Effect of Gao on the cellular activity of Necdin}

Necdin is generally expressed in post-mitotic cells, and implicated in various cellular responses, such as cell growth suppression and neuronal differentiation [25-27]. To determine whether Goo augments the growth sup- pressor activity of Necdin, we performed a BrdU incorporation assay in $293 \mathrm{~T}$ cells expressing various types of $\mathrm{G} \alpha$ and/or FLAG-Necdin plasmids, indicated. To identify transfected cells, we co-transfected with the green fluorescence protein (GFP) expression plasmid, pEGFP. Cells were observed using fluorescence microscopy, and the proportion of BrdU-positive S-phase cells among GFPpositive cells determined (Additional file 1: Figure S1 and Figure 4). G $\alpha$ alone (regardless of the types) did not affect the BrdU-positive cell population, compared to control levels. As expected, expression of Necdin promoted a significant decrease in the proportion of BrdU-positive 

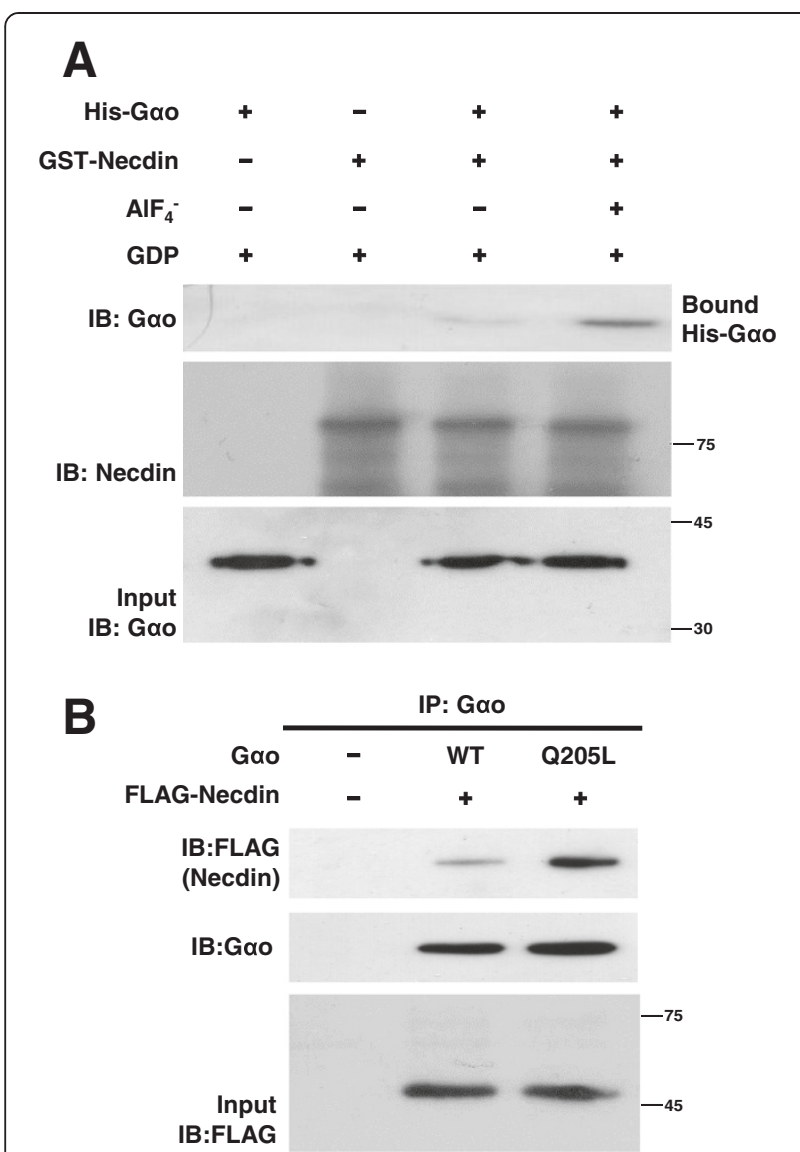

Figure $\mathbf{2}$ Necdin interacts directly and preferentially with activated Gao. (A) His-Gao and GST-Necdin fusion proteins were purified from bacterial lysates. His-Gao (600 nM) was incubated with $30 \mu \mathrm{M}$ GDP andr $\mathrm{AlF}_{4}^{-}$(mixture of $10 \mathrm{mM} \mathrm{NaF}$ and $30 \mu \mathrm{M} \mathrm{AlCl}$ ), as indicated, for $1 \mathrm{~h}$ at $30^{\circ} \mathrm{C}$. Purified GST-Necdin $(200 \mathrm{nM})$ was added to the reaction mixture and incubated for an additional $20 \mathrm{~min}$ at $20^{\circ} \mathrm{C}$. Glutathione-sepharose $4 \mathrm{~B}$ beads were added to the final reaction mixtures, and the beads analyzed by immunoblot analysis using antibodies against Gao and Necdin. Input was loaded with $10 \%$ of His-Gao used for the assay. The numbers beside blot indicate size marker ( $\mathrm{kDa}$ ). (B) Lysates of 293T cells expressing plasmids either GaoWT (3 $\mu \mathrm{g})$ or GaoQ205L $(3 \mu \mathrm{g})$ together with FLAG-Necdin $(10 \mu \mathrm{g})$ were incubated with anti-Gao antibodies. Immunocomplexes were analyzed with antibodies against FLAG and Gao. Input was loaded with 10\% 293T cell extracts used for the immunoprecipitation assay.

cells. Importantly, cell growth suppression induced by Necdin was augmented by the active form of Goo, but not $\mathrm{G \alpha o} / \mathrm{i}$.

Next, we examined whether Goo affects Necdinmediated neuronal differentiation. Neuro2a cells expressing the indicated types of Goo and/or FLAG-Necdin were differentiated via serum starvation. To identify transfected cells, we co-transfected with GFP expression plasmid. At $30 \mathrm{~h}$ after serum starvation, cells were observed under a fluorescence microscope, and the percentage of neurite-bearing cells among the GFP-positive population counted (Additional file 2: Figure S2 and Figure 5A).

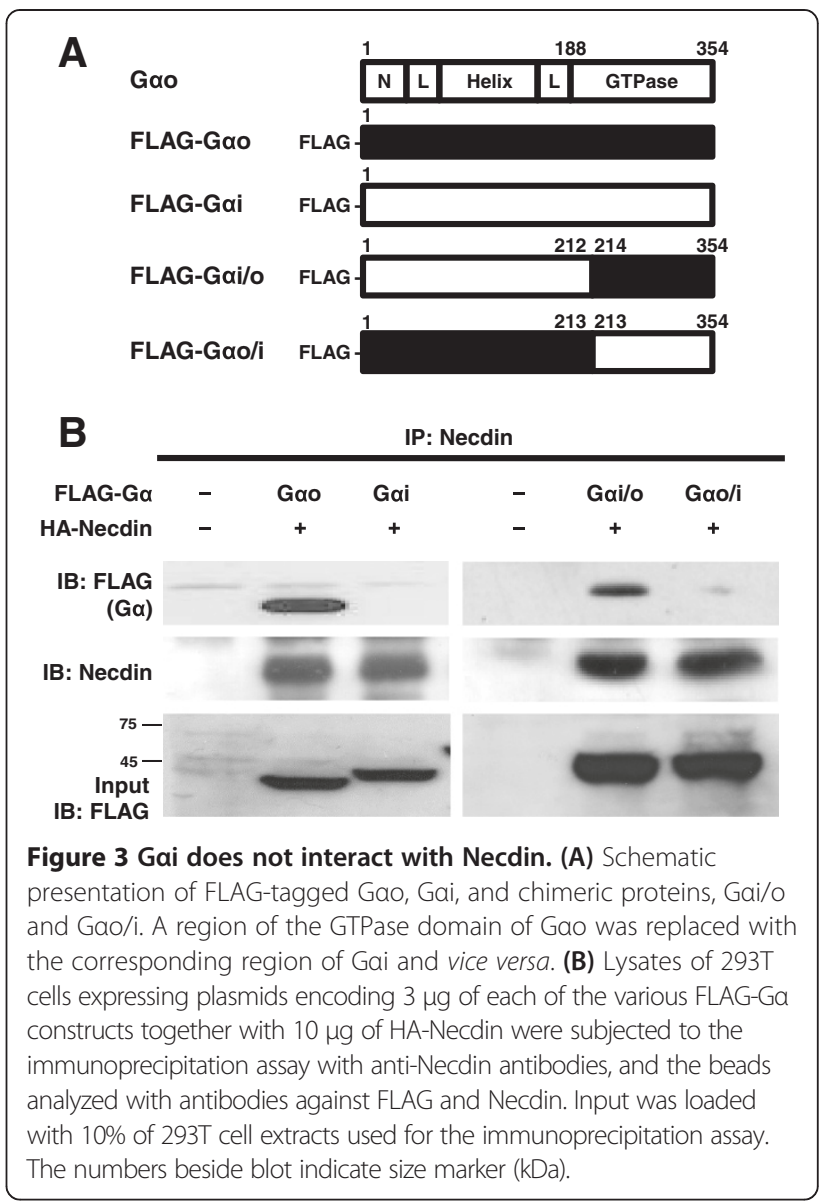

Expression of wild-type and active forms of Goo promoted neurite outgrowth, whereas Goo/i did not induce neurite outgrowth. As expected, Necdin promoted neuronal differentiation in the absence of Goo. Moreover, this effect was synergistically increased by Goo, but not Goo/i expression.

Necdin functions by interacting directly with and antagonizing the function of E2F1, a major cell cycle regulatory protein [22,23]. To determine whether Goo promotes Necdin::E2F1 binding in Neuro2a, we performed an immunoprecipitation assay using lysates of cells expressing plasmid encoding epitope-tagged Necdin, E2F1 and the indicated types of Goo (Figure 5B). Immunocomplexes precipitated with antibodies against FLAG or Necdin were analyzed via immunoblot analysis. Interestingly, interactions between Necdin and E2F1 were significantly increased in the presence of Goo, but not Goo/i. Next, we investigated whether Goo enhances the transcription activity of E2F1. Neuro2a cells were transfected with plasmids encoding FLAG-Necdin and different types of Goo, together with E2F1 and E2F4B-luciferase reporter gene, which contains the E2F1 binding site upstream of the luciferase gene. Luciferase reporter gene activity was increased in the presence of E2F1, and expression of FLAG-Necdin led to a significant decrease in the 


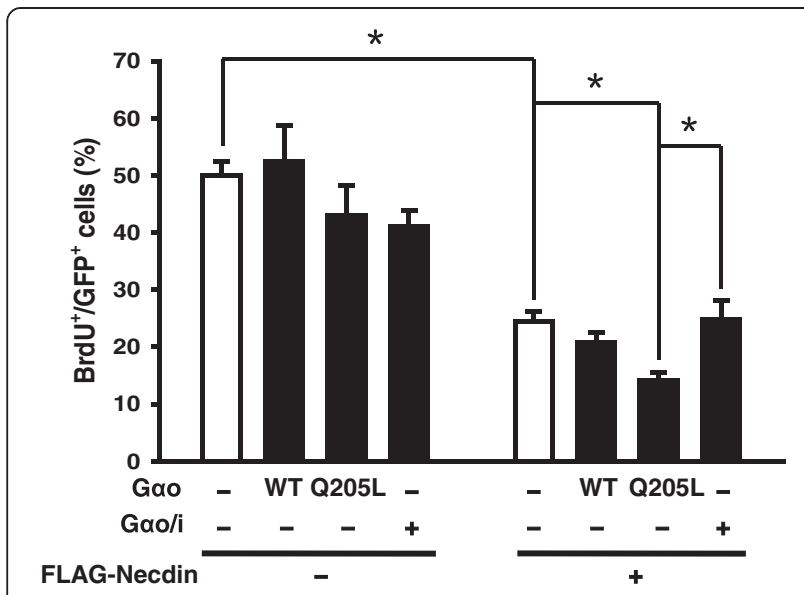

Figure $\mathbf{4}$ Gao enhances cell growth suppression induced by Necdin. 293T cells were transfected with plasmids encoding various types of Ga $(0.5 \mu \mathrm{g})$ and FLAG-Necdin $(1 \mu \mathrm{g})$. To identify transfected cells, we co-transfected with the pEGFP (100 ng). After $24 \mathrm{~h}$ of transfection, cells were labeled with $10 \mu \mathrm{M}$ BrdU for $12 \mathrm{~h}$ and stained with antibodies against BrdU and GFP. The extent of BrdU incorporation was assessed in GFP-positive cells. Data are shown as the average \pm SE of at least three independent experiments. WT and Q205L indicate wild-type and constitutively active mutant of Gao, respectively. ${ }^{*}, p<0.001$.

transcription activity of E2F1, which was synergistically amplified upon co-expression of constitutively activated mutant form of Goo (GooQ205L) (Additional file 3: Figure S3 and Figure 5C). However, Goo/i did not alter the effect of Necdin. Our data clearly indicate that Goo acts as an enhancer of Necdin activity, supporting the theory that Necdin is a candidate downstream effector for Goo.

\section{Effect of CB1R activation on growth suppression in U87MG cells}

We further investigated the effects of Goo-Necdin-E2F1 signaling on growth suppression in U87MG cells endogenously expressing Goo, Necdin, and E2F1. CB1R was additionally expressed as a normal component in cells, and its activation induced the Gi/Go protein-mediated signaling pathway [28]. We examined whether cannabinoid receptor activation increases cell growth suppression and whether this effect is mediated by Goo-Necdin-E2F1 signaling. We investigated the population of S-phase progression cells using a BrdU incorporation assay. Notably, the number of BrdU-positive cells was decreased in the presence of Hu210. This marked repression was recovered upon pretreatment with PTX, Gi/oo inhibitor (Figure 6A), indicative of Gi/Go protein mediation. Using immunoprecipitation analysis, we additionally examined whether Win 55,212-2, CB1R agonist, affects interactions between Necdin and E2F1, and whether these interactions are blocked by PTX pretreatment. We used Win 55,212-2 instead of Hu210 to activate CB1R. In the presence of
Win 55,212-2, we observed Necdin binding to E2F1, which was completely inhibited upon pretreatment with PTX (Figure 6B). The effect of the cannabinoid agonist on Necdin-mediated transcriptional activity of E2F1 was investigated in U87MG cells transfected with the E2F4B-luciferase reporter gene. Treatment with Hu210 induced a dose-dependent reduction in luciferase activity, which was completely abolished upon pretreatment with PTX (Figure 6C). We next tested whether knockdown of Necdin induces recovery of transcriptional activity of E2F1 reduced by CB1R activation. shRNA for Necdin (Necdin-shRNA) expression significantly decrease Necdin expression in U87MG cells (Figure 6D). As shown in Figure 6E, treatment with Win 55,212-2 inhibited transcriptional activity of E2F1 in dose-dependent manner, which was completely abolished by Necdin-shRNA expression. The results indicate that CB1R facilitates U87MG cell growth suppression through the Goo-Necdin-E2F1 signaling pathway.

\section{Discussion}

In the current investigation, Necdin was identified as a novel binding partner of Goo. Interactions between Nectin and Goo were observed both in vitro and in vivo. Moreover, a region within the C-terminal GTPase domain of Goo appears to contribute to this interaction. Interestingly, despite high sequence homology of the GTPase domains of Gai and Goo, Goi did not interact with Necdin. Necdin also bound directly to the active form of Goo with higher affinity than wild-type protein. Accordingly, we propose that Necdin is a candidate downstream effector for Goo.

To further ascertain whether Necdin acts as a functional effector of Goo, experiments were performed using two different cell types: (1) 293T and Neuro2a cells transfected

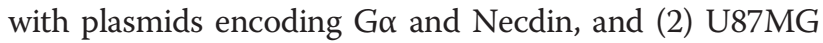
cells endogenously expressing CB1R, which triggers the Gi/o-mediated signaling pathway upon activation. In transfection experiments, the cellular activities of Necdin were significantly enhanced by Goo, but not Goo/i. As shown in Figures 4 and 5, Necdin-induced inhibition of cell proliferation and activation of neuronal differentiation were enhanced synergistically by Goo. Expression of Goo also led to increased Necdin-E2F1 interactions, representative of Necdin activity. In U87MG cells, activated CB1R inhibited cell proliferation via a PTX-sensitive mechanism, meaning Gi/oo-dependency. Cannabinoid additionally activated Necdin-mediated signaling, including NecdinE2F1 interactions and E2F1-mediated transcriptional repression. The results collectively suggest that Necdin functions as a downstream effector for Goo. Nonetheless, the function of Necdin is much less understood in Gprotein signaling. We cannot rule out whether Necdin binds to active Goo thereby prolonging $G \beta \gamma$ signaling. However, the finding that expression of active mutant 

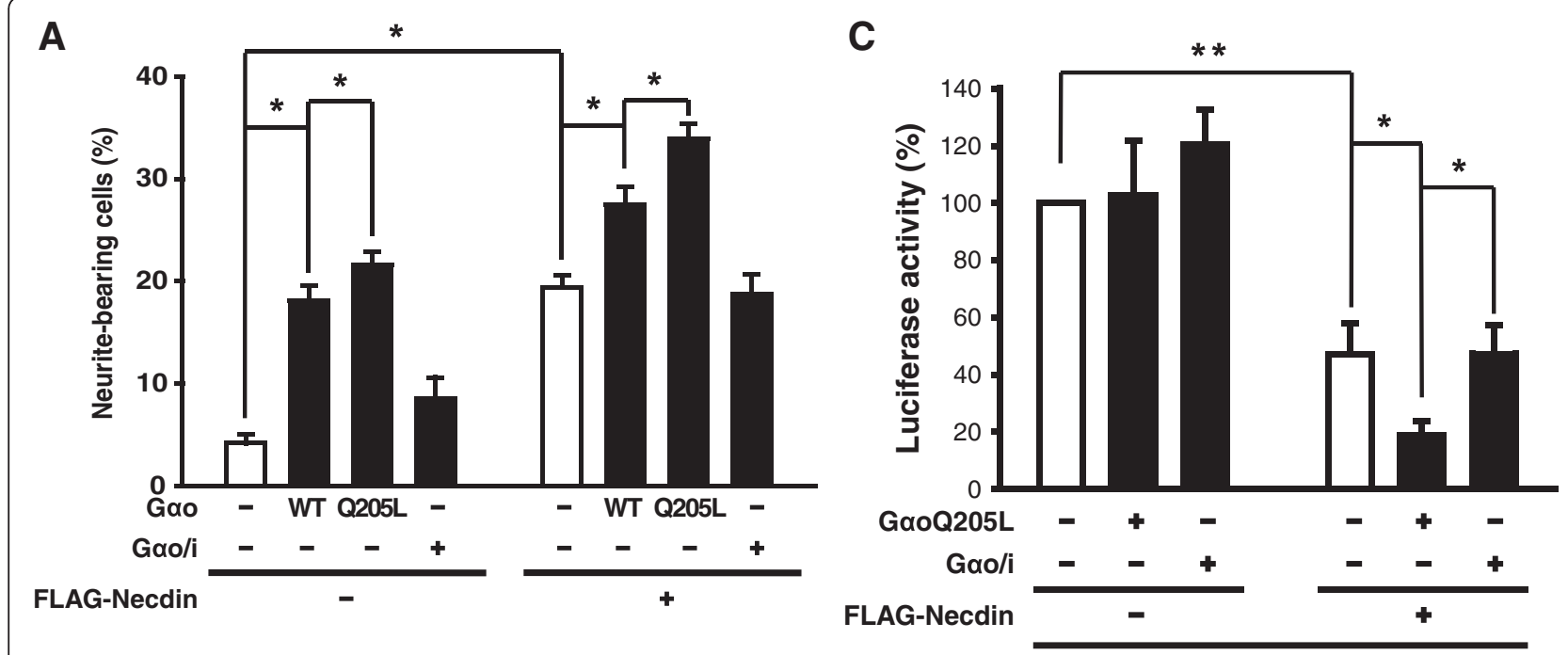

B

IP: FLAG (Necdin)

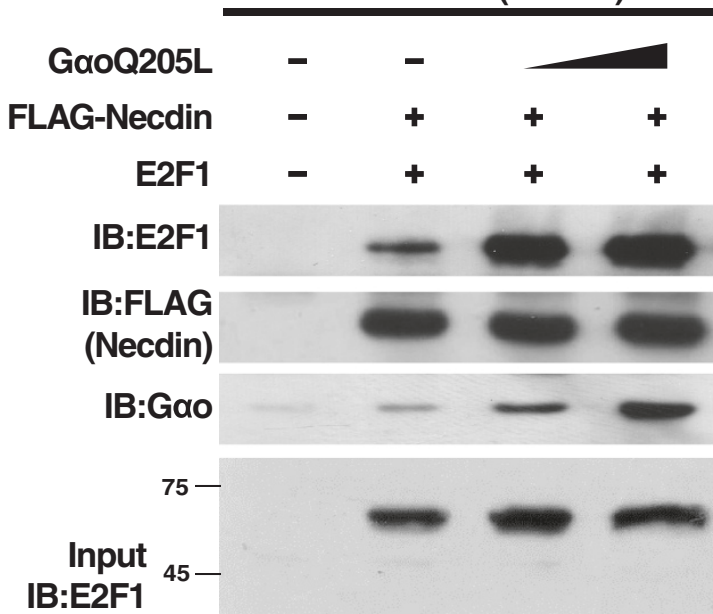

IP: Necdin

$\begin{array}{lllll}- & - & + & - & \text { FLAG-GaoWT } \\ - & - & - & + & \text { FLAG-Gao/i } \\ - & + & + & + & \text { HA-Necdin } \\ - & + & + & + & \text { E2F1 } \\ & 1 & & & \text { IB:E2F1 }\end{array}$

IB:Necdin

IB:FLAG(Ga)

$-75$

-45 Input

IB:E2F1

Figure 5 Gao promotes Necdin-induced neurite outgrowth. (A) Neuro2a cells were transfected with plasmids encoding various types of Ga $(0.5 \mu \mathrm{g})$ and FLAG-Necdin $(1 \mu \mathrm{g})$. To identify transfected cells, we co-transfected with the pEGFP $(100 \mathrm{ng})$. After $24 \mathrm{~h}$ of transfection, cells were serum-starved and observed $30 \mathrm{~h}$ later. The proportions of neurite-bearing cells were determined, as described in Materials and Methods section Data are presented as the average \pm SE of at least three independent experiments. ${ }^{*}, p<0.001$ (B) Left panel, Lysates of Neuro2a cells expressing plasmids encoding FLAG-Necdin $(10 \mu \mathrm{g})$, E2F1 $(0.3 \mu \mathrm{g})$, and GaoQ205L $(1 \mu \mathrm{g}$ or $3 \mu \mathrm{g})$ were subjected to an immunoprecipitation assay using anti-FLAG antibodies, and the beads analyzed with antibodies against E2F1, FLAG, and Gao. Right panel, Neuro2a cells were transfected with plasmids encoding FLAG-GaWT $(10 \mu \mathrm{g})$, HA-Necdin $(20 \mu \mathrm{g})$, and E2F1 $(1 \mu \mathrm{g})$. Cell lysates were immunoprecipitated with anti-Necdin antibodies. Immunocomplexes were subjected to immunoblot analysis using antibodies against E2F1, Necdin, and FLAG. Input was loaded with 10\% of Neuro2a cell extracts used for the immunoprecipitation assay. The numbers beside blot indicate size marker $(\mathrm{kDa})$. (C) Neuro2a cells were transfected with the indicated combinations of plasmids encoding GaoQ205L (0.2 $\mu \mathrm{g})$, FLAG-Gao/i $(0.2 \mu \mathrm{g})$, FLAG-Necdin $(0.25 \mu \mathrm{g})$, E2F1 (0.03 $\mu \mathrm{g})$, E2F4B-Luc reporter gene $(0.1 \mu \mathrm{g})$, and $\beta$-galactosidase $(0.3 \mu \mathrm{g})$. The total amount of plasmid DNA used for transfection was maintained by adding pcDNA3. After $48 \mathrm{~h}$, cells were subjected to luciferase and $\beta$-galactosidase assays. Luciferase activity was normalized to that of $\beta$-galactosidase. Data are presented as the average \pm SE of at least three independent experiments. ${ }^{*}, p<0.05 ;{ }^{* *}, p<0.001$.

of Goo promoted Necdin-mediated cellular activities (Figures 4 and 5) suggests, at least at a first approximation, that Goo can modulate Necdin functions. Further experiments will be needed to evaluate this using $G \beta \gamma$ inhibitor.

Several lines of evidence support the finding that Goo modulates neuronal differentiation. Goo is the most abundant $\mathrm{G} \alpha$ protein in brain tissue and one of the major membrane components of growth cones $[4,29,30]$. Moreover, Goo is sufficient to enhance neuritogenesis in neurogenic cell lines, including PC12, N1E-115 and Neuro2A [5,31]. Goi/o activation induced by CB1R activation inhibits Rap1GAP and activates STAT3 phosphorylation, which, in turn, induces neurite outgrowth in Neuo2A cells 


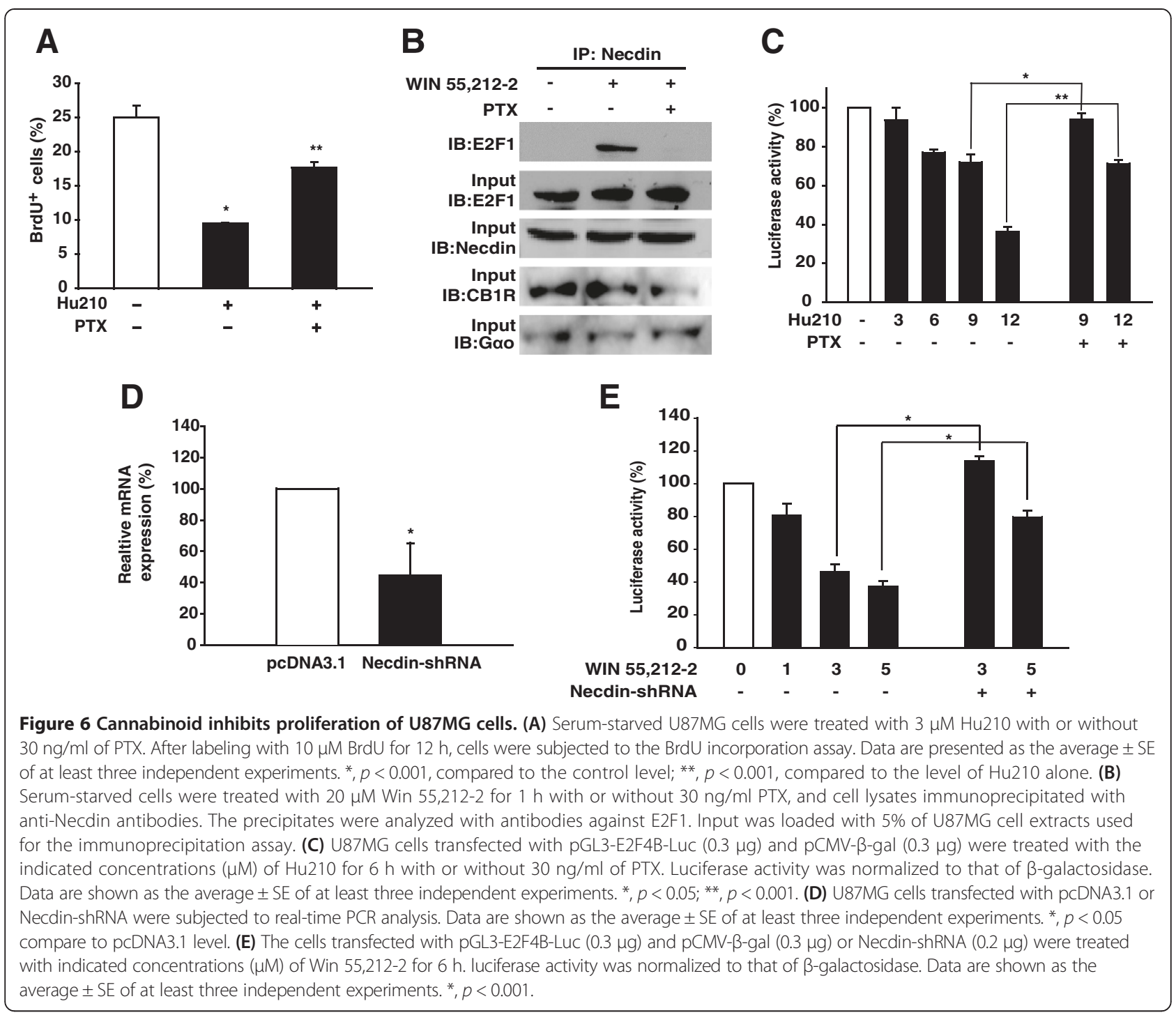

[32]. Consistently, our previous studies demonstrated that Goo functions as an inducer of neuronal differentiation. Goo overexpression led to new neurite formation via modulation of protein kinase A (PKA) signaling in the F11 neuroblastoma cell line [6]. Further experiments showed that Goo attenuates nuclear translocation of the catalytic subunit of PKA via direct interactions and augments its cytosolic effects [24]. Additionally, Goo stimulated the activity of Rit, which induced Erk-mediated neuronal differentiation in Neuro2a cells [7]. Therefore, Goo mediates signaling of neuronal differentiation by mobilizing various downstream signaling molecules including Necdin.

We postulate that Necdin-mediated neuronal differentiation is attributable to its activity as a strong growth suppressor. Necdin exhibits anti-proliferative activity in various cells and is highly expressed in normal tissues but downregulated in tumor cells [26,33]. Necdin expression is suppressed by STAT3 whose activation promotes tumor development [34-36]. Constitutive expression of STAT3 induces a decrease in Necdin expression at the mRNA and protein levels in tumor cell lines [37]. A previous study demonstrated that a constitutively active form of Goo induces STAT3-mediated NIH-3 T3 cell transformation [38]. Thus, we considered the possibility that Goo activation induces cell transformation by enhancing STAT3 activity, in turn, inhibiting Necdin expression. However, in contrast to previous findings, expression of Goo alone did not affect proliferation in $293 \mathrm{~T}$ cells (Figure 4). This discrepancy may be explained by the fact that STAT3 activation is an uncommon signaling pathway for Goo activation in 293T cells, compared to NIH-3 T3 cells.

Considerable evidence supports the theory that cannabinoids are potential therapeutic agents for cancer. Cannabinoids have been shown to inhibit tumor cell proliferation in various cell types, including glioma, pheochromocytoma, 
prostate, and leukemia cells [39-42]. Cannabinoids also inhibit tumor development by blocking angiogenesis and invasiveness $[8,43,44]$. As expected, cannabinoid induced inhibition of cell proliferation in our study. Importantly, cannabinoid activated Necdin-mediated signaling in a PTX-sensitive manner. Although CB1R is coupled with

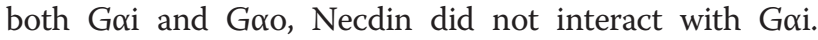
Therefore, we propose that the CB1R-Necdin signaling pathway is mediated by Goo, independently of Goi. The anticancer effects of cannabinoid are mediated via several signaling pathways [45]. We speculate that together with previously reported signaling pathways, the CB1R-GooNecdin-E2F1 pathway contributes to inhibition of cell proliferation in U87MG cells.

Several assay systems are available to measure the cellu-

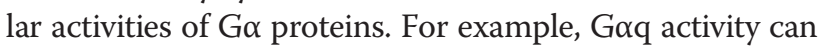
be measured based on accumulation of inositol phosphate, which is modulated by phospholipase $\mathrm{C}$, a downstream effector of Goq. The amount of accumulated cAMP represents the activity of adenylyl cyclase, a downstream effector of Gai and Gos. However, an effective assay system to measure Goo activity has not been developed to date owing to the ambiguity of its downstream effectors. Our results showing that Necdin is a candidate downstream effector of Goo independent of Goi may facilitate the development of a Necdin-specific assay system for Goo.

\section{Conclusions}

We have identified a novel downstream effector molecule and signaling pathway for Goo. Activation of Goo enhances Necdin-E2F1 interactions, which, in turn, modulate cell differentiation and proliferation. Our current findings present an additional novel signaling pathway to explain the diverse roles of Goo and cannabinoid receptor.

\section{Methods}

\section{Construction of plasmids}

The pRC/CMV-Necdin plasmid was a generous gift from Dr. K. Yoshikawa (Osaka University, Japan). pcDNA3E2F1 and pGL3-E2F4B-Luc reporter plasmids were kindly provided by Dr. J. Cheong (Pusan National University, Korea). Plasmids pRC/CMV-GooWT and pRC/CMV-

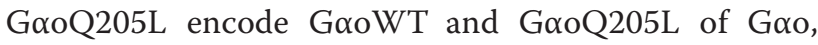
respectively [41]. To generate pcFLAG-Necdin, a FLAGNecdin expression plasmid, pRC/CMV-Necdin, was amplified using the primers 5 '-CAT GTC GGA ACA AAG TAA G-3' and 5'-ATT-TAG-GTG-ACA-CTATAG-3'. Amplified PCR products were inserted into pGEM-T Easy plasmid (Promega, Madison, WI, USA). pcFLAG-Necdin was generated by digesting pGEM-TNecdin with the restriction enzymes, NotI and XbaI, and ligating the fragment into the corresponding sites of pcFLAG. pcHA-Necdin encoding HA-Necdin was constructed by digesting pcFLAG-Necdin with NotI and $\mathrm{XbaI}$ and ligating the fragment into the corresponding restriction sites of pcHA. pGEX-5X-Necdin, an expression plasmid for GST-Necdin, was generated by digesting pcFLAG-Necdin with EcoRI and cloning into the corresponding restriction sites of pGEX-5X (GE Healthcare Life Science, Piscataway, NJ, USA). To generate psi-RNAhH1ZeoG2-Necdin, Necdin-shRNA plasmid, pcFLAGNecdin was amplified using the primers 5'-GTA CCT CGC CCG AAG AAC GGA TAG AAG ATC AAG AGT CTT CTA TCC GTT CTT CGG GCT TTT TGG AAA-3' and 5'-AGC TTT TCC AAA AAG CCC GAA GAA CGG ATA GAA GAC TCT TGA TCT TCT ATG CGT TCT TCG GGC GAG-3'. Amplified PCR products were inserted into psi-RNA-hH1ZeoG2 plasmid (Invivogen, San Diego, CA) using HindIII and ACC65I restriction enzymes.

\section{Yeast two-hybrid screening}

The bait plasmid (pHybTrp/Zeo-GooQ205L) was transformed into the yeast reporter cell line, L40, with the mouse brain cDNA library (Clontech, Palo Alto, CA, USA) as recommended by the manufacturer. The methods used for isolation of positive clones are described in a previous report [41].

\section{Cell culture and transfection}

Human embryonic kidney cell line, 293T, mouse neuroblastoma cell line, Neuro2a, and human glioblastoma cell line, U87MG, were maintained in DMEM supplemented with $10 \%$ fetal bovine serum (FBS), 100 units $/ \mathrm{ml}$ penicillin and $100 \mu \mathrm{g} / \mathrm{ml}$ streptomycin. 293T and Neuro2a cells were transiently transfected with the indicated concentrations of plasmids using calcium phosphate and polyethylenimine, respectively. For affinity binding assays, after $48 \mathrm{~h}$ of transfection, cells were harvested and extracted with PBTX buffer (PBS containing $5 \mathrm{mM} \mathrm{MgCl}_{2}, 1 \mathrm{mM}$ EDTA, $1 \%$ Triton $\mathrm{X}-100,5 \mu \mathrm{g} / \mathrm{ml}$ aprotinin, $10 \mu \mathrm{g} / \mathrm{ml}$ leupeptin, $2 \mu \mathrm{g} / \mathrm{ml}$ pepstatin $\mathrm{A}$, and $2 \mathrm{mM}$ phenylmethylsulfonyl fluoride) for $1 \mathrm{~h}$ at $4^{\circ} \mathrm{C}$ with gentle rotation.

\section{GST pulldown assay}

BL21 bacterial cells transformed with pGEX2T-Goo plasmids [24] encoding GST-Goo fusion proteins were induced with $0.1 \mathrm{mM}$ IPTG and lysed using a standard protocol. Lysates were incubated with glutathione-Sepharose $4 \mathrm{~B}$ beads (GE Healthcare Life Science) in PBTX (total volume of $500 \mu \mathrm{l}$ ) for $1 \mathrm{~h}$ at $4^{\circ} \mathrm{C}$ with gentle rotation, and the beads washed extensively with PBTX buffer. 293T cell extracts $(500 \mu \mathrm{g})$ expressing $10 \mu \mathrm{g}$ FLAG-Necdin were added to GST-Goo-bound beads and incubated for $1 \mathrm{~h}$ at $37^{\circ} \mathrm{C}$ (total volume of $500 \mu \mathrm{l}$ ). After extensive washing with PBTX buffer, bound proteins were eluted with SDS 
sample buffer and subjected to immunoblot analysis with antibodies against FLAG (1:500 dilution, Sigma-Aldrich, St. Louis, MO, USA).

\section{Direct interactions between His-Gao and GST-Necdin}

Fusion proteins, His-Goo and GST-Necdin, were purified from BL21 cells using glutathione-Sepharose $4 \mathrm{~B}$ beads and HisTrap $^{\mathrm{TM}}$, respectively [41]. His-Goo (600 nM) was incubated with $30 \mu \mathrm{M}$ GDP and $\mathrm{AlF}_{4}^{-}$(mixture of $10 \mathrm{mM}$ $\mathrm{NaF}$ and $30 \mu \mathrm{M} \mathrm{AlCl}$ ), as indicated, in HEMNDL buffer (20 mM Na-HEPES, pH 8.0, 1 mM EDTA, 2 mM MgSO $150 \mathrm{mM} \mathrm{NaCl}, 1 \mathrm{mM}$ dithiothreitol, and $0.05 \%$ Triton X$100)$ for $1 \mathrm{~h}$ at $30^{\circ} \mathrm{C}$. GST-Necdin was added to the reaction at a final concentration of $200 \mathrm{nM}$, and incubated for an additional $20 \mathrm{~min}$ at $20^{\circ} \mathrm{C}$. Following the addition of glutathione-Sepharose $4 \mathrm{~B}$ beads, reactions were incubated for $1 \mathrm{~h}$ at $4^{\circ} \mathrm{C}$ with gentle rotation. Beads charged with proteins were analyzed with the indicated antibodies.

\section{Immunoprecipitation assay}

$293 \mathrm{~T}$ and Neuro2a cells $\left(1.5 \times 10^{6}\right.$ cells/dish $)$ were plated on $100 \mathrm{~mm}$ tissue culture dishes and transiently transfected with the appropriate combinations of expression plasmids. U87MG cells $\left(1 \times 10^{5}\right.$ cells $/$ dish $)$ were plated on $100 \mathrm{~mm}$ tissue culture dishes. After $24 \mathrm{~h}$, cells were serum-starved with $2 \%$ FBS for $20 \mathrm{~h}$. Cells were treated with $20 \mu \mathrm{M}$ Win 55,212-2, a CB1R agonist, for $1 \mathrm{~h}$ in the absence or presence of $30 \mathrm{ng} / \mathrm{ml}$ pertussis toxin (PTX, Calbiochem, La Jolla, CA, USA), a Gi/oo inhibitor. Pretreatment with PTX was performed for $20 \mathrm{~h}$ prior to Win 55,212-2 application. Newborn rat brain extracts were prepared as reported previously [24]. Cell or brain extracts were pre-cleared by incubating with $20 \mu$ Protein A-Sepharose CL-4B beads (10\% slurry) (GE Healthcare Life Science) for $20 \mathrm{~min}$, followed by the addition of $1 \mu \mathrm{g}$ of the indicated antibodies with gentle rotation for $4 \mathrm{~h}$ at $37^{\circ} \mathrm{C}$, and subsequently, $50 \mu \mathrm{l}$ beads. After $2 \mathrm{~h}$ of incubation at $4^{\circ} \mathrm{C}$, beads were washed with PBTX. Bound proteins were subjected to immunoblot analysis using antibodies against FLAG, Goo (1:1000, Santa Cruz Biotechnology, Santa Cruz, CA, USA), Necdin (1:500, Cayman Chemicals, Ann Arbor, MI, USA), and E2F1 (1:200, Santa Cruz Biotechnology).

\section{Immunofluorescence assay}

293 T cells $\left(1 \times 10^{3}\right.$ cells/coverslip) were plated on coverslips and transfected with expression plasmids for Goo $(0.1 \mu \mathrm{g})$ and FLAG-Necdin $(0.25 \mu \mathrm{g})$. After $48 \mathrm{~h}$, cells were fixed with $4 \%$ paraformaldehyde in PBS for $15 \mathrm{~min}$ and incubated for $1 \mathrm{~h}$ at room temperature with antibodies against Goo and FLAG. Cells were washed with PBS and incubated with Alexa Fluor 488-conjugated goat anti-rabbit IgG and Alexa Fluor 568-conjugated goat anti-mouse IgG (Life Technologies, Gaithersburg,
MD, USA) for $30 \mathrm{~min}$ at room temperature. After washing with PBS and counterstaining with DAPI (Vector Laboratories, Burlingame, CA, USA), cells were observed under a LSM510 confocal laser scanning microscope (Carl Zeiss, Thornwood, NY, USA).

\section{Tissue preparation and immunohistochemistry}

Pregnant mice (C57BL/6 J) were killed via cervical dislocation and E15.5 embryos were placed immediately in ice-cold 4\% PFA. After overnight fixation, tissues were embedded in paraffin and sectioned to $5-\mu \mathrm{m}$ thickness. Antigens were retrieved by boiling in $10 \mathrm{mM}$ sodium citrate ( $\mathrm{pH}$ 6.0) in a microwave oven, the sections were blocked in PBS with 5\% normal serum, and probed with primary antibodies against Necdin (1:100; Santa Cruz Biotechnology) and Goo (1:400). The sections were then incubated with Alexa Fluor 488- or 594-conjugated antiIgG secondary antibodies and counterstained with Hoechst 33258 (Molecular Probes, Eugene, OR). Fluorescent images were acquired using a Zeiss LSM710 confocal microscope (Carl Zeiss).

\section{Bromodeoxyuridine (BrdU) incorporation assay}

293T and U87MG cells were plated on 6-well plates at a density of $2 \times 10^{4}$ cells per well. $293 \mathrm{~T}$ cells were transfected with the appropriate combinations of indicated plasmids. To identify transfected cells, we co-transfected with the GFP expression plasmid in all experiments. U87MG cells were serum-starved and pretreated with $30 \mathrm{ng} / \mathrm{ml}$ PTX. After $20 \mathrm{~h}$, cells were treated with $3 \mu \mathrm{M}$ Hu210 (Tocris Bioscience, Ellisville, Mo, USA), a CB1R agonist for $6 \mathrm{~h}$. Both cell lines were labeled with $10 \mu \mathrm{M}$ BrdU (Sigma-Aldrich), $12 \mathrm{~h}$ before harvest. Next, cells were washed with PBS and fixed in $4 \%$ paraformaldehyde in PBS for $15 \mathrm{~m}$. After fixation, cells were subjected to immunofluorescence staining against BrdU [46].

\section{Neurite outgrowth assay}

Neuro2a cells were plated on 6-well tissue culture dishes at a density of $3 \times 10^{4}$ cells per well. Cells were transfected with the indicated combinations of plasmids, and $100 \mathrm{ng}$ of pEGFP. After $24 \mathrm{~h}$, cells were serum-starved for $36 \mathrm{~h}$. Fluorescence images were observed with a IX71 fluorescence microscope (Olympus, Tokyo, Japan), and the percentage of neurite-bearing cells among the GFP-positive population calculated. Cell processes greater than cell body in length were counted as neurites.

\section{Real-time PCR}

Total RNA was extracted from cultured cells with the Easy-spin total RNA extraction kit (Intron Biotechnology, Sungnam, Korea), according to the manufacturer's instructions. First-strand complementary DNA (cDNA) was synthesized using $1 \mu \mathrm{g}$ of total RNA as the template, 
500 ng of oligo (dT), and AccuPower ${ }^{\text {tm }}$ RT-Premix (Bioneer, Daejeon, Korea) in a total volume of $20 \mu \mathrm{l}$, according to the manufacturer's recommendation. The relative mRNA expression of Necdin was assessed using TOPreal $^{\text {tw }}$ qPCR premix (Enzyomics, Daejeon, Korea) on the CFX96 $6^{\text {tw }}$ PCR System (Bio-Rad, Richmond, CA). Primer sequences used were as follows: Necdin, 5'-GCT CAT GTG GTA CGT GTT GG-3'; and 5'-TGC TTC TGC ACC ATT TCT TG-3'; GAPDH, 5'-TGG GCT ACA CTG AGC ACC AG-3'; and 5'-GGG TGT CGC TGT TGA AGT CA-3'. The Necdin mRNA values were normalized to the amount of GAPDH that was measured.

\section{Luciferase reporter gene assay}

Neuro2a cells were plated on 6-well tissue culture dishes at a density of $3 \times 10^{4}$ cells per well. Cells were transfected with the indicated combination of plasmids, together with $0.1 \mu \mathrm{g}$ of pGL3-E2F4B-Luc reporter plasmids containing four consensus E2F binding sites [47]. For normalization of transfection efficiency, cells were transfected with $0.3 \mu \mathrm{g}$ of $\beta$-galactosidase expression plasmid (pCMV$\beta$-gal). The total amount of plasmid DNA used for transfection was maintained by adding pcDNA3 (Life Technologies). After $48 \mathrm{~h}$, cell lysates were assayed for luciferase and $\beta$-gal activity using the Luciferase Assay System (Promega, Madison, WI, USA), as recommended by the manufacturer. U87MG cells were plated on 6-well plates at a density of $1 \times 10^{4}$ cells per well and transfected with $0.3 \mu \mathrm{g}$ of pGL3-E2F4B-Luc reporter plasmids using Lipofectamine 2000 (Life Technologies). After 24 h, cells were serum-starved for $16 \mathrm{~h}$ and treated with the indicated amounts of Hu210 for $6 \mathrm{~h}$ in the absence or presence of $30 \mathrm{ng} / \mathrm{ml}$ PTX. Pretreatment with PTX was performed for $1 \mathrm{~h}$ before Hu210 application. For knockdown experiment, U87MG cells transfected with pGL3-E2F4B-Luc $(0.3 \mu \mathrm{g})$ and Necdin-shRNA $(0.2 \mu \mathrm{g})$ were treated with indicated amount of Win 55,212-2 for $6 \mathrm{~h}$.

\section{Additional files}

Additional file 1: Figure S1. Gao enhances cell growth suppression induced by Necdin. $293 \mathrm{~T}$ cells were transfected with plasmids encoding various types of $\mathrm{Ga}(0.5 \mu \mathrm{g})$ and FLAG-Necdin $(1 \mu \mathrm{g})$, as indicated. To identify transfected cells, we co-transfected with the pEGFP (100 ng). After $24 \mathrm{~h}$ of transfection, cells were labeled with $10 \mu \mathrm{M}$ BrdU for $12 \mathrm{~h}$ and stained with antibodies against BrdU and GFP. Scale bar, $20 \mu \mathrm{m}$.

Additional file 2: Figure S2. Gao promotes Necdin-induced neurite outgrowth. Neuro2a cells were transfected with plasmids encoding various types of Ga $(0.5 \mu \mathrm{g})$ and FLAG-Necdin $(1 \mu \mathrm{g})$. To identify transfected cells, we co-transfected with the pEGFP (100 ng). After $24 \mathrm{~h}$ of transfection, cells were serum-starved and observed $30 \mathrm{~h}$ later. Scale bar, $50 \mu \mathrm{m}$.

Additional file 3: Figure S3. Effect of E2F1 and Necdin on E2F4Bluciferase reporter gene activity. Neuroza cells were transfected with the indicated combinations of plasmids encoding FLAG-Necdin $(0.25 \mu \mathrm{g})$ E2F1 $(0.03 \mu \mathrm{g})$, E2F4B-Luc reporter gene $(0.1 \mu \mathrm{g})$, and $\beta$-galactosidase $(0.3 \mu \mathrm{g})$. The total amount of plasmid DNA used for transfection was maintained by adding pcDNA3. After $48 \mathrm{~h}$, cells were subjected to luciferase and $\beta$-galactosidase assays. Luciferase activity was normalized to that of $\beta$-galactosidase. Data are presented as the average \pm SE of at least three independent experiments. ${ }^{*}, p<0.001$.

\section{Abbreviations}

BrdU: Bromodeoxyuridine; CB1R: Type 1 cannabinoid receptor; CB2R: Type II cannabinoid receptor; FBS: Fetal bovine serum; Gao: alpha subunit of Go; GaoQ205L: Constitutively activated mutant of Gao; GaoWT: Wild-type of Gao; GFP: Green fluorescence protein; GPCR: G protein-coupled receptors; GST: Glutathione-S-transferase; Necdin: Neurally differentiated embryonal carcinoma-derived protein; PKA: Protein kinase A; PTX: Pertussis toxin; Rit: Ras-like protein in all tissues; Rb: Retinoblastoma protein.

\section{Competing interests}

The authors declare that they have no competing interests.

\section{Authors' contributions}

HJ, SL, SK and SG designed experiment, HJ, SL and SK performed experiments, and SG and HJ wrote the manuscript. All authors read and approved the final manuscript.

\section{Acknowledgements}

We thank Dr. K. Yoshikawa (Osaka University, Japan) for the Necdin plasmids and Dr. J. Cheong (Pusan National University, Korea) for E2F1 and E2F4B-Luc reporter plasmids. This research was supported by the Basic Science Research Program through the National Research Foundation of Korea (NRF) funded by the Ministry of Education, Science and Technology (NRF-2012R1A1A2005207 to SG).

\section{Author details}

'Department of Life Science, Kyonggi University, Suwon 443-760, Republic of Korea. ${ }^{2}$ Department of Anatomy, Ajou University, School of Medicine, Suwon 443-721, Republic of Korea.

Received: 27 January 2014 Accepted: 12 June 2014

Published: 10 July 2014

\section{References}

1. Gilman AG: G proteins: transducers of receptor-generated signals. Annu Rev Biochem 1987, 56:615-649.

2. Baltoumas FA, Theodoropoulou MC, Hamodrakas SJ: Interactions of the alpha-subunits of heterotrimeric G-proteins with GPCRs, effectors and RGS proteins: a critical review and analysis of interacting surfaces, conformational shifts, structural diversity and electrostatic potentials. J Struct Biol 2013, 182:209-218.

3. Sternweis PC, Robishaw JD: Isolation of two proteins with high affinity for guanine nucleotides from membranes of bovine brain. J Biol Chem 1984, 259:13806-13813

4. Strittmatter SM, Valenzuela D, Kennedy TE, Neer EJ, Fishman MC: G0 is a major growth cone protein subject to regulation by GAP-43. Nature 1990, 344:836-841.

5. Jordan JD, He JC, Eungdamrong NJ, Gomes I, Ali W, Nguyen T, Bivona TG, Philips $M R$, Devi LA, lyengar R: Cannabinoid receptor-induced neurite outgrowth is mediated by Rap1 activation through $\mathrm{G}($ alpha)o/i-triggered proteasomal degradation of Rap1GAPII. J Biol Chem 2005, 280:11413-11421.

6. Ghil SH, Kim BJ, Lee YD, Suh-Kim H: Neurite outgrowth induced by cyclic AMP can be modulated by the alpha subunit of Go. J Neurochem 2000, 74:151-158.

7. Kim SH, Kim S, Ghil SH: Rit contributes to neurite outgrowth triggered by the alpha subunit of Go. Neuroreport 2008, 19:521-525.

8. Casanova ML, Blazquez C, Martinez-Palacio J, Villanueva C, FernandezAcenero MJ, Huffman JW, Jorcano JL, Guzman M: Inhibition of skin tumor growth and angiogenesis in vivo by activation of cannabinoid receptors. J Clin Invest 2003, 111:43-50.

9. Aquado T, Monory K, Palazuelos J, Stella N, Cravatt B, Lutz B, Marsicano G, Kokaia Z, Guzman M, Galve-Roperh I: The endocannabinoid system drives neural progenitor proliferation. FASEB J 2005, 19:1704-1706.

10. Julien B, Grenard P, Teixeira-Clerc F, Van Nhieu JT, Li L, Karsak M, Zimmer A, Mallat A, Lotersztajn S: Antifibrogenic role of the cannabinoid receptor CB2 in the liver. Gastroenterology 2005, 128:742-755. 
11. Galve-Roperh I, Aguado T, Palazuelos J, Guzman M: The endocannabinoid system and neurogenesis in health and disease. Neuroscientist 2007, 13:109-114.

12. Pan HL, Wu ZZ, Zhou HY, Chen SR, Zhang HM, Li DP: Modulation of pain transmission by G-protein-coupled receptors. Pharmacol Ther 2008, 117:141-161.

13. Matsuda LA, Lolait SJ, Brownstein MJ, Young AC, Bonner TI: Structure of a cannabinoid receptor and functional expression of the cloned cDNA. Nature 1990, 346:561-564.

14. Kaminski NE, Abood ME, Kessler FK, Martin BR, Schatz AR: Identification of a functionally relevant cannabinoid receptor on mouse spleen cells that is involved in cannabinoid-mediated immune modulation. Mol Pharmacol 1992, 42:736-742.

15. Munro S, Thomas KL, Abu-Shaar M: Molecular characterization of a peripheral receptor for cannabinoids. Nature 1993, 365:61-65.

16. Bouaboula M, Poinot-Chazel C, Marchand J, Canat X, Bourrie B, Rinaldi-Carmona M, Calandra B, Le Fur G, Casellas P: Signaling pathway associated with stimulation of CB2 peripheral cannabinoid receptor. Involvement of both mitogen-activated protein kinase and induction of Krox-24 expression. Eur J Biochem 1996, 237:704-711.

17. Maruyama K, Usami M, Aizawa T, Yoshikawa K: A novel brain-specific mRNA encoding nuclear protein (necdin) expressed in neurally differentiated embryonal carcinoma cells. Biochem Biophys Res Commun 1991, 178:291-296.

18. Taniura H, Taniguchi N, Hara M, Yoshikawa K: Necdin, a postmitotic neuron-specific growth suppressor, interacts with viral transforming proteins and cellular transcription factor E2F1. J Biol Chem 1998, 273:720-728.

19. Kuwajima T, Nishimura I, Yoshikawa K: Necdin promotes GABAergic neuron differentiation in cooperation with Dlx homeodomain proteins. J Neurosci 2006, 26:5383-5392.

20. Fujiwara K, Hasegawa K, Ohkumo T, Miyoshi H, Tseng YH, Yoshikawa K: Necdin controls proliferation of white adipocyte progenitor cells. PLOS One 2012, 7:e30948.

21. Pessina P, Conti V, Tonlorenzi R, Touvier T, Meneveri R, Cossu G, Brunelli S: Necdin enhances muscle reconstitution of dystrophic muscle by vessel-associated progenitors, by promoting cell survival and myogenic differentiation. Cell Death Differ 2012, 19:827-838.

22. Kuwako K, Taniura H, Yoshikawa K: Necdin-related MAGE proteins differentially interact with the E2F1 transcription factor and the p75 neurotrophin receptor. J Biol Chem 2004, 279:1703-1712.

23. Kobayashi M, Taniura H, Yoshikawa K: Ectopic expression of necdin induces differentiation of mouse neuroblastoma cells. J Biol Chem 2002, 277:42128-42135.

24. Ghil S, Choi JM, Kim SS, Lee YD, Liao Y, Birnbaumer L, Suh-Kim H: Compartmentalization of protein kinase A signaling by the heterotrimeric G protein Go. Proc Natl Acad Sci U S A 2006, 103:19158-19163.

25. Taniura $H$, Kobayashi M, Yoshikawa K: Functional domains of necdin for protein-protein interaction, nuclear matrix targeting, and cell growth suppression. J Cell Biochem 2005, 94:804-815.

26. Andrieu D, Meziane H, Marly F, Angelats C, Fernandez PA, Muscatelli F: Sensory defects in Necdin deficient mice result from a loss of sensory neurons correlated within an increase of developmental programmed cell death. BMC Dev Biol 2006, 6:56

27. Francois S, D'Orlando C, Fatone T, Touvier T, Pessina P, Meneveri R, Brunelli $\mathrm{S}$ : Necdin enhances myoblasts survival by facilitating the degradation of the mediator of apoptosis CCAR1/CARP1. PLoS One 2012, 7:e43335

28. Aguado T, Romero E, Monory K, Palazuelos J, Sendtner M, Marsicano G, Lutz B, Guzman M, Galve-Roperh I: The CB1 cannabinoid receptor mediates excitotoxicity-induced neural progenitor proliferation and neurogenesis. J Biol Chem 2007, 282:23892-23898.

29. Tsukamoto T, Toyama R, Itoh H, Kozasa T, Matsuoka M, Kaziro Y: Structure of the human gene and two rat cDNAs encoding the alpha chain of GTP-binding regulatory protein Go: two different mRNAs are generated by alternative splicing. Proc Natl Acad Sci U S A 1991, 88:2974-2978.

30. Strittmatter SM, Vartanian T, Fishman MC: GAP-43 as a plasticity protein in neuronal form and repair. J Neurobiol 1992, 23:507-520.

31. Strittmatter SM, Fishman MC, Zhu XP: Activated mutants of the alpha subunit of $\mathrm{G}(\mathrm{o})$ promote an increased number of neurites per cell. J Neurosci 1994, 14:2327-2338.

32. He JC, Gomes I, Nguyen T, Jayaram G, Ram PT, Devi LA, lyengar R: The G alpha(o/i)-coupled cannabinoid receptor-mediated neurite outgrowth involves Rap regulation of Src and Stat3. J Biol Chem 2005, 280:33426-33434.

33. De Faveri LE, Hurst CD, Platt FM, Taylor CF, Roulson JA, Sanchez-Carbayo M, Knowles MA, Chapman EJ: Putative tumour suppressor gene necdin is hypermethylated and mutated in human cancer. Br J Cancer 2013, 108:1368-1377.

34. Grandis JR, Drenning SD, Chakraborty A, Zhou MY, Zeng Q, Pitt AS, Tweardy DJ: Requirement of Stat3 but not Stat1 activation for epidermal growth factor receptor- mediated cell growth In vitro. J Clin Invest 1998, 102:1385-1392.

35. Catlett-Falcone R, Landowski TH, Oshiro MM, Turkson J, Levitzki A, Savino R, Ciliberto G, Moscinski L, Fernandez-Luna JL, Nunez G, Dalton WS, Jove R: Constitutive activation of Stat3 signaling confers resistance to apoptosis in human U266 myeloma cells. Immunity 1999, 10:105-115.

36. Zammarchi F, de Stanchina E, Bournazou E, Supakorndej T, Martires K, Riedel E, Corben AD, Bromberg JF, Cartegni L: Antitumorigenic potential of STAT3 alternative splicing modulation. Proc Natl Acad Sci U S A 2011 108:17779-17784.

37. Haviland R, Eschrich S, Bloom G, Ma Y, Minton S, Jove R, Cress WD: Necdin, a negative growth regulator, is a novel STAT3 target gene down-regulated in human cancer. PLoS One 2011, 6:e24923.

38. Ram PT, Horvath CM, lyengar R: Stat3-mediated transformation of NIH-3 T3 cells by the constitutively active Q205L Galphao protein. Science 2000, 287:142-144.

39. Velasco G, Galve-Roperh I, Sanchez C, Blazquez C, Guzman M: Hypothesis: cannabinoid therapy for the treatment of gliomas? Neuropharmacology 2004, 47:315-323.

40. Sarfaraz S, Afaq F, Adhami VM, Mukhtar H: Cannabinoid receptor as a novel target for the treatment of prostate cancer. Cancer Res 2005, 65:1635-1641.

41. Won JH, Park JS, Ju HH, Kim S, Suh-Kim H, Ghil SH: The alpha subunit of Go interacts with promyelocytic leukemia zinc finger protein and modulates its functions. Cell Signal 2008, 20:884-891.

42. Gurley SN, Abidi AH, Allison P, Guan P, Duntsch C, Robertson JH, Kosanke SD, Keir ST, Bigner DD, Elberger AJ, Moore BM 2nd: Mechanism of anti-glioma activity and in vivo efficacy of the cannabinoid ligand KM-233. J Neurooncol 2012, 110:163-177

43. Portella G, Laezza C, Laccetti P, De Petrocellis L, Di Marzo V, Bifulco M: Inhibitory effects of cannabinoid CB1 receptor stimulation on tumor growth and metastatic spreading: actions on signals involved in angiogenesis and metastasis. FASEB J 2003, 17:1771-1773.

44. Blazquez C, Carracedo A, Barrado L, Real PJ, Fernandez-Luna JL, Velasco G, Malumbres M, Guzman M: Cannabinoid receptors as novel targets for the treatment of melanoma. FASEB J 2006, 20:2633-2635.

45. Pisanti S, Picardi P, D'Alessandro A, Laezza C, Bifulco M: The endocannabinoid signaling system in cancer. Trends Pharmacol Sci 2013, 34:273-282.

46. Won JH, Ghil SH: The GTPase domain of Galphao contributes to the functional interaction of Galphao with the promyelocytic leukemia zinc finger protein. Cell Mol Biol Lett 2009, 14:46-56.

47. Kong HJ, Yu HJ, Hong S, Park MJ, Choi YH, An WG, Lee JW, Cheong J: Interaction and functional cooperation of the cancer-amplified transcriptional coactivator activating signal cointegrator-2 and E2F-1 in cell proliferation. Mol Cancer Res 2003, 1:948-958.

\section{doi:10.1186/s12964-014-0039-9}

Cite this article as: Ju et al:: The alpha subunit of Go modulates cell proliferation and differentiation through interactions with Necdin. Cell Communication and Signaling 2014 12:39. 